\title{
Traumatismos Craneanos Fetales en el Parto en Presentación de Pelvis
}

\author{
Dr. Saulo Muñoz Delgado - Dr. Gerardo Chadid Samur \\ Clínica de Maternidad de Cali
}

\begin{abstract}
El análisis de los traumatismos fetales en el parto en presentación de pelvis es delicado pues coloca al obstetra y a la institueión en una situación difícil ya que se pueden desprender severas críticas para ambos.
\end{abstract}

Solamente un estudio sereno de la mortalidad fetal en el parto en presentación de pelvis puede mostrar el mecanismo de producción de estos traumatismos y obtener una correlación clínica patológica.

El reconocimiento valeroso y oportuno de Ridlez (1926) sobre la menor mortalidad en los partos de pelvis atendidos por una escuela de comadronas, al ser comparada con la obtenida por los médicos, dio las normas que se siguen hoy en día sobre reducción en las maniobras en la conducción de estos partos.

En el mecanismo del parto en presentación de pelvis se pueden presentar serias anomalías que hacen necesarias intervenciones y maniobras las cuales van a influír sobre el pronóstico fetal.

Existen diversas escuelas con múltiples maniobras para resolver estas situaciones las cuales no entramos a analizar, limitándonos a buscar la relación que existe entre los partos intervenidos y las lesiones fetales.

En los partos en presentación de vértice la cabeza va sufriendo un moldeamiento progresivo con el fin de adaptar los mayores diámetros del polo cefálico en el sentido del eje de progresión de la pelvis materna. No sucede lo mismo en la presentación de pel- 
vis en donde la cabeza última para su expulsión sufre bruscamente esta adaptación, que surrada a maniobras mal conducidas aumenta notablemente la mortalidad fetal.

Como en el curso de estos partos se produce frecuentemente anoxia, la cual es también un traumatismo del parto, queremos aclarar que consideramos como traumatismo únicamente a aquel "limitado a lesión mecánica local que produce gran hemorragia". (1)

Nuestro interés es solamente presentar los hallazgos de autopsia en un número de casos atendidos durante los 4 últimos años por personal en diversas etapas de preparación y estudiarlos para evitar en un futuro su repetición.

\section{MATERIAL Y METODOS}

Presentamos los resultados de autopsia de 18 casos de niños nacidos en la Clínica de Maternidad de Cali en presentación de pelvis desde el año de 1955 hasta septiembre de 1959.

Las autopsias fueron practicadas por los miembros del Departamento de Patología de la Universidad del Valle quienes utilizan la técnica de Beneke (3) para la apertura de la cavidad craneana, técnica que presenta la ventaja de conservar la integridad de los senos venosos y dar campo suficiente para una buena exploración del sistema vascular y las meninges, evitando falsas interpretaciones por artificios de técnica.

Las lesiones las hemos dividido en dos grupos: lesiones vasculares y lesiones meníngeas.

En todos los casos se hizo el diagnóstico final anatomo-patológico y correlación clínico-patológica.

\section{RESULTADOS}

La incidencia de presentación de pelvis en la Clínica de Maternidad es similar a los demás centros, promediando $(3,3 \%)$.

(Ver Cuadro No 1 ). 


\section{Cuadro No. 1}

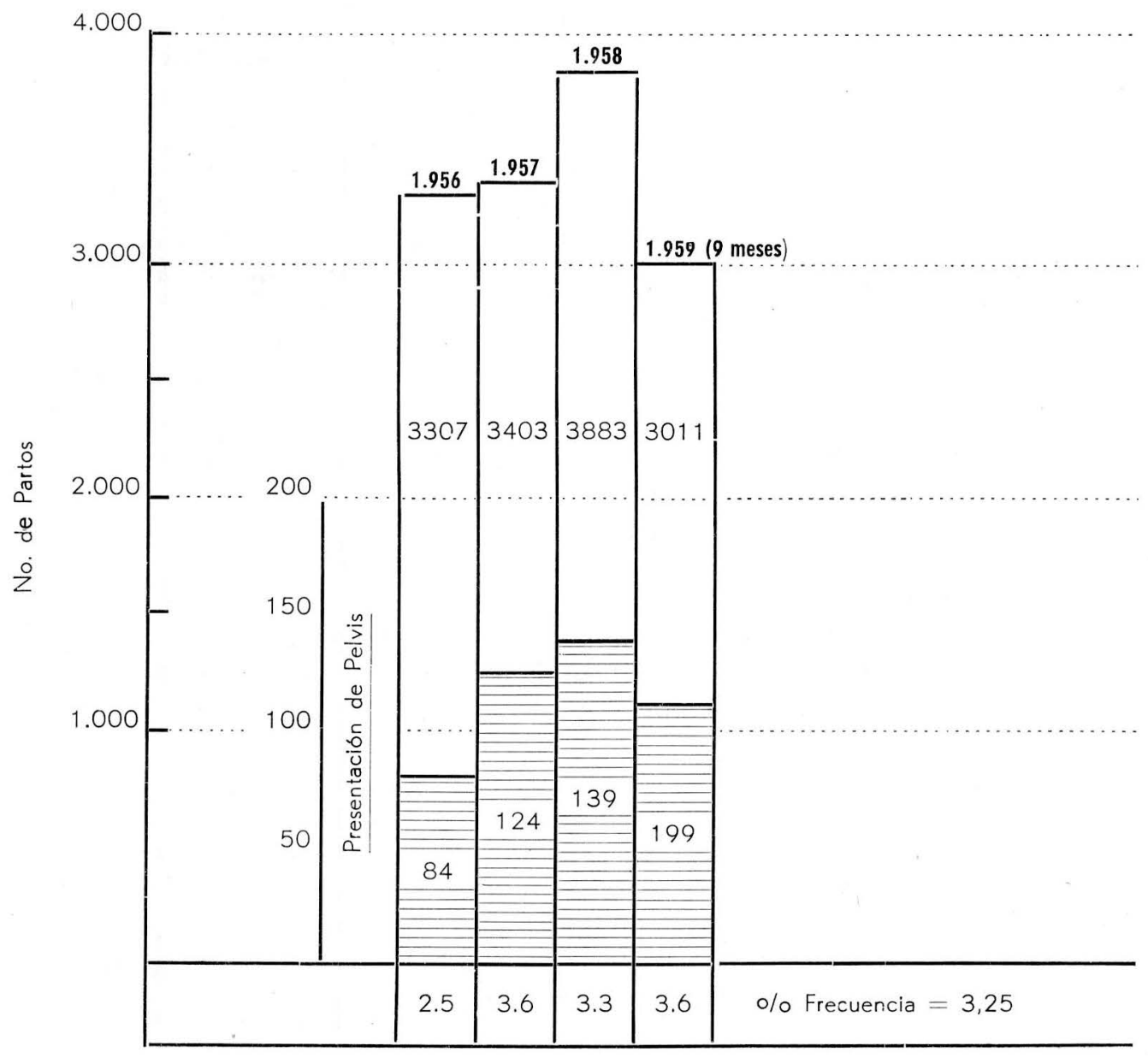

FRECUENCIA EN LA PRESENTACION DE PELVIS

El análisis de la mortalidad global en los 4 años demuestra una reducción a la mitad de la mortalidad en el último año, descendiendo del $30.9 \%$ al $14.6 \%$.

(Ver Cuadro $\mathrm{N}^{\circ}$ 2).

La lesión vascular más frecuente fue hemorragia subaracnoidea, la cual se presentó en 11 de los 18 casos, en la gran mayoría consecutiva a desgarros meníngeos. Le sigue la ruptura de la vena de Galeno, que la encontramos en 5 casos. 
Cuadro No. 2

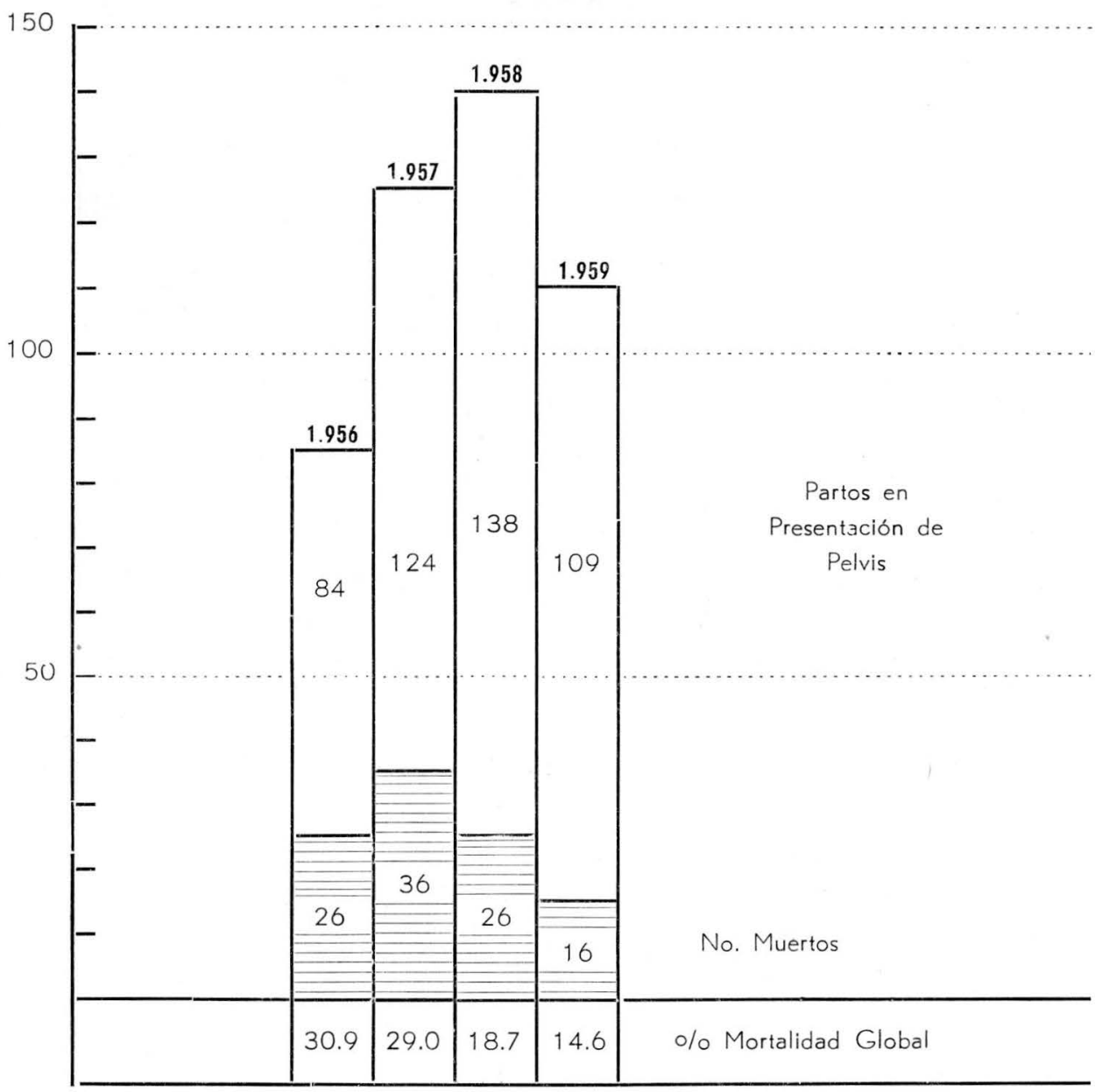

\section{MORTALIDAD FETA.L - PRESENTACION PELVIS}

La ruptura del seno longitudinal se acompañó de lesión de las meninges en 2 de los casos. También encontramos un cefalohematoma el cual se debe considerar traumático ya que es el único mecanismo de producción en el parto de pelvis. (Ver Cuadro No 3 ).

Las lesiones meníngeas regularmente asociadas con lesiones vasculares son más frecuentes en la tienda del cerebelo. En nues- 


\section{HALLASGOS ANATOMO-PATOLOGICOS}

Lesiones Vasculares

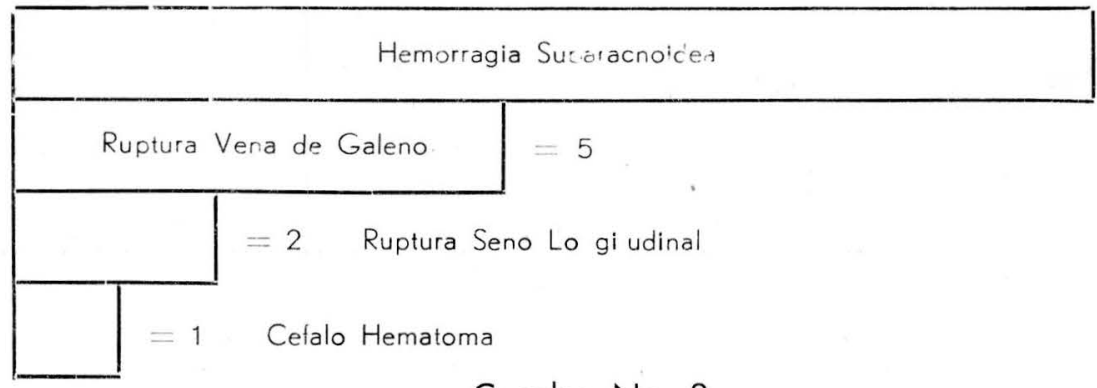

Cuadro No. 3

tra observación se presentó en 5 casos. La hoz del cerebro se lesionó en 3 casos y se encontró ruptura de las meninges en diversos sitios en 1 caso.

(Ver Cuadro $\mathrm{N}^{0} 4$ ).

Lesiones Meningeas

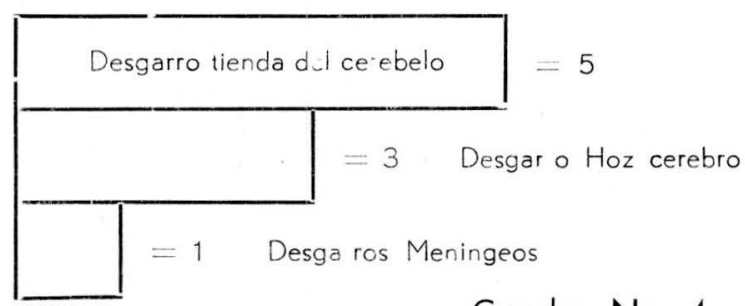

Cuadro No. 4

De los 18 casos autopsiados en 13 se practicaron maniobras e intervenciones tales como extracción podálica, maniobra de Mauriceau, forceps en cabeza última, etc. Los 13 casos anteriores presentaron lesiones craneanas de tipo traumático. En los casos en que se produjo el parto espontáneo, la causa de muerte fue distinta a las lesiones de tipo traumático.

El peso de los niños varió de 1.200 a $4.600 \mathrm{gms}$. (Ver Cuadros Nos. 5 y 6 ). 


\section{CORRELACION CLINICO/PATOLOGICA}

Cuadros No. 5 y No.6

\begin{tabular}{|c|c|c|c|c|c|}
\hline CASO No. & PESO & INTERVENCION & $\begin{array}{c}\text { LESIONES } \\
\text { VASCULARES }\end{array}$ & $\begin{array}{l}\text { LESIONES DE } \\
\text { MENINGES }\end{array}$ & CAUSA DE MUERTE \\
\hline 1 & 1.640 & Ext. podálica & Sí & Sí & Hem. cerebral \\
\hline 2 & 2.400 & $\begin{array}{l}\text { Ext. podálica } \\
\text { Forceps }\end{array}$ & Sí & Sí & Hem. cerebral \\
\hline 3 & 2.600 & Ext. podálica + & Sí & Sí & Hem. cerebral \\
\hline 4 & 2.600 & $\begin{array}{l}\text { Versión y } \\
\text { Ext. podálica }+\end{array}$ & Si & Sí & Hem. cerebral \\
\hline 5 & 3.200 & $\begin{array}{l}\text { Ext. podálica } \\
\text { Mauriceau }\end{array}$ & Sí & Sí & Hem. cerebral \\
\hline 6 & 3.200 & Ext. podálica & Sí & Sí & Hem. cerebral \\
\hline 7 & 3.740 & $\begin{array}{l}\text { Versión y } \\
\text { Ext. podálica }\end{array}$ & Si & Sí & Hem. cerebral \\
\hline 8 & 3.860 & Ext. podálica + & No & No & $\begin{array}{l}\text { Anoxia por pro- } \\
\text { lapso de cordón }\end{array}$ \\
\hline 9 & 4.600 & $\begin{array}{l}\text { Ext. podálica } \\
\text { Mauriceau }\end{array}$ & Sí & Sí & Hem. cerebral \\
\hline 10 & 1.740 & Mauriceau & No & No & $\begin{array}{l}\text { Peritonitis } \\
\text { Septicemia }\end{array}$ \\
\hline 11 & 2.400 & Mauriceau & Sí & Sí & Hem. cerebral \\
\hline 12 & 2.480 & Mauriceau & Sí & Sí & Hem. cerebral \\
\hline 13 & 3.580 & Mauriceau & Si & Sí & Hem. cerebral \\
\hline 14 & 1.200 & No intervenido & No & No & $\begin{array}{l}\text { Atelectasia } \\
\text { pulmonar }\end{array}$ \\
\hline 15 & 1.400 & No intervenido & No & No & $\begin{array}{l}\text { Atelectasia } \\
\text { pulmonar }\end{array}$ \\
\hline 16 & 1.650 & No intervenido & No & No & $\begin{array}{l}\text { Anoxia por pro- } \\
\text { lapso de cordón }\end{array}$ \\
\hline 17 & 2.500 & No intervenido & No & No & Neumonía \\
\hline 18 & 3.160 & No intervenido & No & No & $\begin{array}{l}\text { Atelectasia } \\
\text { pulmonar }\end{array}$ \\
\hline
\end{tabular}




\section{COMENTARIOS}

La mortalidad global fetal en parto en presentación de pelvis, nos muestra en el último año una reducción del $30.9 \%$ al $14.6 \%$. Al analizar esta reducción hemos encontrado los factores que han influído sobre ésta y que clasificamos según su importancia:

1) Mejor evaluación clínica de la pelvis materna y del tamaño fetal.

2) Mejor conocimiento del mecanismo de parto en presentación de pelvis y de la forma en que las maniobras producen los traumatismos.

3) Abstención de interferencias mientras el parto progrese normalmente.

4) Reducción de la versión y gran extracción podálica a sus indicaciones absolutas.

5) Bloqueo pudendo y aplicación de forceps de Piper como maniobra selectiva en cabeza última.

6) Atención del parto por 2 ó más miembros bien entrenados del personal del servicio.

Aunque las lesiones se han dividido para su descripción en vasculares y meníngeas, éstas últimas llevan en la mayoría de los casos a lesiones vasculares. Durante el trabajo de parto en presentación de vértice, el polo cefálico sufre una reducción de sus diámetros (moldeamiento) el cual se produce por reducción del diámetro suboccipitobregmático con aumento del diámetro occipito-mentoniano. Este moldeamiento se hace mediante presiones graduales las cuales se transmiten a la parte media de las meninges, distribuyéndose simétricamente a ambos lados de los hemisferios, evitando en esta forma el que se rompan las meninges, la hoz del cerebro o la tienda del cerebelo (1). En realidad la ruptura de las meninges no tendría significación si no estuviesen íntimamente relacionadas con las estructuras vasculares. Su ruptura lesiona los vasos produciendo hemorragia que por hipertensión 
endocraneana determina la muerte. Así vemos con frecuencia que al desgarrarse la tienda del cerebelo se rompe la vena de Galeno, la cual está situada en la confluencia de las venas internas de ambos hemisferios, con una longitud de $1 \mathrm{~cm}$. y desemboca en el seno recto. Cuando se produce un aumento anormal del diámetro antero-posterior de la cabeza se pone en tensión exagerada la vena de Galeno y si las presiones aumentan bruscamente se produce su ruptura. Si durante la tensión de las venas se ejercen tracciones en el eje longitudinal, se desgarra más fácilmente.

Un mecanismo similar sucede al desgarrarse la hoz del cerebro, produciéndose la ruptura del seno longitudinal.

En los casos en que encontramos hemorragia subaracnoidea, aun cuando ésta es de tipo anóxico, en nuestros casos estaba asociada a lesiones vasculares o meníngeas.

\section{CONCLUSIONES}

1) La mayor mortalidad se debió a maniobras traumáticas que ocasionaron lesiones vasculares cerebrales y desgarros meníngeos.

2) Se puede obtener una mayor reducción de la mortalidad fetai en el parto en presentación de pelvis mediante la observación de las normas anteriormente definidas.

3) Para un correcto diagnóstico de la causa de muerte fetal en el parto en presentación de pelvis es indispensable la práctica de autopsia.

\section{PIBLIOGRAFIA}

1 POTTER E. L.: Pathology of the fetus and the nowborn. The year book publishers. Illinois 1957. pp. 84-95.

2 LEON J.: Presentación pelviana. Librería "El Ateneo". Editorial. Buenos Aires, 1953.

3 MUÑOZ S. M., FERNANDEZ H. Forceps de Piper. Método electivo en presentación de pelvis. Rev. Col. Obst. y Ginec. 9: 65, 1958. 